Alejandro Piscitelli ${ }^{1}$ / piscitelli. alejandro@gmail.com
1 Licenciado en Filosofía en la Universidad de Buenos Aires, Master en Ciencias de Sistemas en la Universidad de Louisville (Estados Unidos) y Master en Ciencias Sociales por la FLACSO (Argentina). Se desempeña como Profesor Titular del Taller de Procesamiento de Datos, Telemática e Informática, en la carrera de Ciencias de la Comunicación, UBA. También enseña en FLACSO y en la Universidad de San Andrés.

Fecha de recepción: 31 de octubre de 2013 / Fecha de aprobación: 8 de noviembre de 2013

\section{¿Cómo definir a las Humanidades Digitales? ¿0 no definirlas?}

\section{Resumen}

Desde una postura crítica, en el presente artículo se busca definir las humanidades digitales, entendiéndolas a partir de una perspectiva que aunque contempla su relación con la tecnología, busca dar un sentido a lo humano en esta era, partiendo de la consideración de lo representativo en el contexto digital y su vínculo con el desarrollo del pensamiento en la red. Desde allí, se analiza el papel de la educación que amerita intervenciones mínimamente invasivas pero de máximo poder. El cierre de estas reflexiones se hace en relación con los nuevos lenguajes y por extensión las nuevas competencias e inmersiones que éstos hacen posibles y que resultan de sumo interés para las intervenciones propuestas.

Palabras clave: humanidades digitales, humanistas digitales, sistema educativo en la era digital, pedagogía de la enunciación, pedagogía de la sorpresa.

\section{How to define the Digital Humanities? Or not define?}

\section{Summary}

From a critical stance in the present article seeks to define digital humanities, understanding them from a perspective that even contemplates his relationship with technology, seeking to make sense of the human in this era, from the consideration of the representative in the digital environment and its link to the development of thought in the network. From there, the role of education that merits minimally invasive interventions but maximum power is analyzed. The closure of these reflections is done in relation to new languages and by extension the new skills and dives they make possible and which are of great interest for the proposed interventions.

Key words: digital humanities, digital humanists, education system in the digital age, the enunciation pedagogy, pedagogy of surprise.

Keywords: digital humanities, digital humanists, education system in the digital age, the enunciation pedagogy, pedagogy of surprise.

\section{Como definir os Humanidades Digitais? Ou não define?}

\section{Resumo}

A partir de uma postura crítica no presente artigo procura definir humanidades digitais, compreendê-los a partir de uma perspectiva que contempla ainda a sua relação com a tecnologia, buscando dar sentido ao ser humano nesta época, a partir da consideração do representante em ambiente digital e sua ligação com o desenvolvimento do pensamento na rede. A partir daí, o papel da educação que merece intervenções minimamente invasivas, mas a potência máxima é analisada. 0 fechamento destas reflexões é feita em relação a novas linguagens e, por extensão, as novas competências e mergulhos fazem possivel e que são de grande interesse para as intervenções propostas.

Palavras-chave: humanidades digitais, humanistas digital, sistema de educação na era digital, a pedagogia, pedagogia enunciação de surpresa. 


\section{Desde la computación humanística hacia las humanidades digitales}

A pesar de que últimamente se ha publicado más de una decena de libros seminales y de compilaciones sobre las $\mathrm{Hu}$ manidades Digitales, es difícil llegar a un consenso sobre en qué consisten. Como bien señala Rafael Alvarado (2011), en vez de una definición, tenemos una genealogía, una red de parentesco de familias entre escuelas de pensamiento, intereses metodológi$\cos y$ herramientas preferidas. Es decir, una historia de personas que han decidido autodenominarse como humanistas digitales, $\mathrm{y}$ que en el proceso de definir el término lo están recreando, como corresponde a toda definición generativa. Se trata de una categoría social, no de una ontológica.

Parafraseando a Matthew Kirschenbaum, uno de los padres fundadores de la disciplina, las DH (con sus iniciales en inglés), giran alrededor de una revista cuya referencia es Digital Humanities, una oficina nacional en USA, una conferencia anual, y una red internacional de centros académicos bajo el auspicio de la Alliance of Digital Humanities Organizations (ADHO).

El desplazamiento de las actividades desde la computación humanística hacía las humanidades digitales, muestra un notable crecimiento en la cantidad de personas, instituciones y publicaciones. Contrario a lo que ha sucedido durante muchos años, cuando sus promotores se conformaban con asistir a microscópicos THATCamp, como ocurrió en Buenos Aires hace un mes; esto quiere decir que mientras en otras latitudes hay multitudes interesadas y dispuestas a abordar el tema, en Latinoamérica aún no se llega a este nivel.

\section{Una variedad inagotable de enfoques y procedimientos}

Las novedades que ofrece el enfoque humanista digital, también permiten afirmar que se presentan distintas perspectivas a tener en cuenta, de acuerdo con la diversidad de grupos de trabajo que se ocupan del tema:

- Los que descartan los “remanentes textuales", es decir, aquellos que anulan las cualidades propias de las humanidades analógicas y van más allá del texto, apartándose del conocimiento visto como una "aplicación basada en los textos", cuyos curadores son los críticos literarios, los historiadores o los bibliotecarios.

- Los que han abrazado las humanidades espaciales a través del uso del software que mapea el texto sobre la visualización de la geografía humana.

- Críticos estadísticos, que van desde el Padre Roberto Busa y su alianza con IBM, hasta Franco Moretti y su alianza con NGram de Google, quienes insisten en que la aplicación de métodos de lingüística computacional y la observación de grandes corpus, harán posible la concepción de hipótesis inaccesibles a la mera lectura (distant reading).

- Proyectos como el Critical Code Studies, que interpreta al código fuente como un conjunto de símbolos y procesos a explorar.

Todos estos y muchos otros ejemplos remiten a las humanidades digitales, pero no las definen a pesar de estar entre los proyectos mejor dotados financieramente, contar con muchas ideas y con profesionales reconocidos. Sin embargo, como insiste Alvarado (2011), ninguno de ellos ha hecho una diferencia epistemológica abismal, aunque quizás el problema esté en otro lado, en nuestra incapacidad para definir las disciplinas de un modo que recoja los nuevos postulados y formas de hacer planteados por las humanidades digitales.

Porque convengamos que para un outsider, pero también muchas más veces para insiders críticos, es muy difícil saber la diferencia entre historia, sociología, economía, antropología y arqueología. Esto se debe a que todas estas "disciplinas" tienen más coincidencias que diferencias, y estas últimas se basan, más que en cualquier otra razón, en el modo en que se da cuenta de un dominio particular de datos, su adquisición, organización, análisis e interpretación.

\section{Indefiniendo una disciplina, haciendo cosas al programar}

Para los humanistas digitales, las tecnologías computacionales pueden ser cosas muy diferentes, y el grado de radicalidad de su tarea diferirá de acuerdo con este hecho. No es lo mismo imaginar las tecnologías computacionales como herramientas, como texto o como metáforas. Sin embargo, y para evitar tantas vueltas, podemos convenir en que las humanidades digitales son más que

nada un currículum, un conjunto interrelacionado de dominios y recursos que contribuye a la construcción de conocimiento.

Quizás lo que mejor define las humanidades digitales no sean las tecnologías a las que recurren (innumerables y volátiles), ni los métodos (que provienen de los campos consagrados más diversos), sino cierto "encuentro" alegre y juguetón con la represen- 
tación digital en sí misma. Dicho encuentro implica dos detalles peculiares y muy interesantes: por un lado, que esa representación digital está cambiando a causa de la permanente evolución del software, y por otro, de que en vez de remitirnos a los árboles, los Arrays, los Lattices y otras combinaciones bidimensionales de datos, tenemos la oportunidad de imaginar otras posibilidades tridimensionales cada vez más complejas y ricas.

No menos interesante es que los humanistas digitales son cada vez más ellos mismos programadores y constructores de dispositivos, de herramientas y de plataformas; por lo tanto, pueden participar de un tipo de conversaciones para la acción que se da muy pocas veces en la historia. Los humanistas digitales de última generación forman parte del movimiento $\mathrm{Maker}^{2}$, trabajan en Labs, son bilingües teórico/prácticos, en algunos casos son polimatas ${ }^{3}$; ejercen saberes conceptuales y prácticos, por lo que están en capacidad de combinar el conocimiento y la acción de un modo similar al de los artesanos y filósofos renacentistas. Aunque tamaña ambición sea una exageración, es al mismo tiempo una interesante posibilidad; mientras tanto, como los humanistas digitales hacen más de lo que dicen, podemos ver qué ocurre cuando sus esfuerzos gravitan hacia iniciativas pedagógicas vinculadas a modos nada convencionales de "tratar" la complejidad.

\section{Hacia una educación mínimamente invasiva}

\section{De la apología a la síntesis de la complejidad}

Casi un siglo de lucha contra el reduccionismo y el determinismo llevó a que desde distintos campos se empezara a desarrollar una apología de la complejidad. Ludwig von Bertalanffy (1901-1972) y su teoría general de los sistemas, forma parte de esta pléyade de visionarios y anticipadores, pero sus apuestas y propuestas fueron más que nada una compilación de consejos bien intencionados, antes que instructivos para el cambio puntual. El plano en el que se desplegaron sus intuiciones y anticipaciones fue más bien el filosófico, el epistemológico, anche el metafísico, pero nunca el de las transformaciones en el aquí y ahora.

Pasaron muchas cosas en el siglo XX, tachonado de maravillas y tragedias, antes de que esas admoniciones devinieran en itinerario efectivo a recorrer; de que en vez de consejos se convirtieran en planes de

\section{Hacer es conectar}

Son innumerables las obras que han señalado este territorio en los últimos años, pero de entre ellas, algunas nos sirven de hilo conductor para desarrollar nuestra aldea, que es el mundo entero. A título de inventario resulta útil la revisión de los tex- acción, y que, a diferencia de las retóricas deontológícas, se transmutaran en ciclos de ideación/prototipeado.

Para que esa alquimia tuviera lugar, intervinieron tantos factores que enumerarlos pasteurizaría cualquier inventario, ya que incluyeron dosis de epistemología constructivista, de historia social de la ciencia, de programación y bits, de lectura distante; de neomarxismo y neofreudismo; de memética, de autopoiesis, y de tantas cosas más (que jalonaron nuestra deformación cognitiva, emocional y práxica). Aún así, en el plano simbólico, en el material y en el social, no hay duda de que el factor unificador de tantas iniciativas dispersas, y raramente destinadas a un maridaje feliz, han sido las redes sociales y la conectómica ${ }^{4}$, desarrolladas en el campo de la vida social masivamente digital.

tos de Solé (2009); Christakis y Fowler (2010); Watts (2004), Buchanan (2003) y Laszlo-Barabasi (2002), todos los cuales han vestido de gala cursos sucesivos de la Cátedra de Datos $(\mathrm{UBA})^{5}$, desde 1996 en adelante.

2 Este corresponde a una tendencia de producción de soluciones tecnológicas en las que individuos o grupos no usan la gran infraestructura de diseño y producción de la gran industria, sino que utilizan los recursos de información disponibles en la red, procesos de producción de bajo coste e incluso materiales reciclados. Buena parte de lo producido y de lo que se encuentra en el entorno digital, es de código abierto para que cualquiera acceda, use y cree usando la información disponible.

3 Dominan diversos campos o dominios de conocimiento.

4 Campo de la neurociencia que se ocupa de la construcción de un mapa de la red neuronal. Desde 2009 se desarrolla el proyecto del conectoma humano (en concurencia con el proyecto del genoma humano), cuya pretensión es contar con una representación de la conectividad fisiológica y anatómica del cerebro humano.

5 Espacio de formación ofrecido desde la Facultad de Ciencias Sociales de la Universidad de Buenos Aires; ha tenido en la mira, desde 1996, el estudio y la reflexión permanentes desde los planteamientos seminales de la teoría general de sistemas y la cibernética, hasta la contemporánea discusión de las humanidades digitales. Todo ello desde la perspectiva de la acción y la experimentación transformadora y propositiva; por eso se sustenta en la idea del taller, del laboratorio, para este caso de procesamiento de datos. Mayor información en: http://catedradatos.com.ar/ 
Pero una cosa es leer libros y otra muy distinta es lograr que los libros nos hablen, no sólo, o no tanto, a partir de las palabras escritas, sino de sus instrucciones para diseñar dispositivos que cambien nuestros comportamientos en todos los planos. Desde el más pedestre (pero insondable y rara vez lograble), de hacer que la clase se convierta aquí y ahora en un evento olímpico, pasando por el rediseño institucional (como es el caso de crear Labs de Arte/ Ciencia), hasta llegar a la política misma, cuando nos deshacemos de la cultura de la queja, del aquí no podemos, y de echarle la culpa a no se sabe qué tercero o cuarto, nombrado o no, y nos ponemos finalmente a hacer y a dejar hacer, rediseñándonos en el intento.

Este pasaje al diseño y a la acción tiene un nombre y apellido: La Red. Desde hace 5, 10 o 15 años según la medida que tome-

\section{Del cerebro, sus debilidades y fortalezas}

Pero lo que se conecta en red no es un entelequia, sino un cuerpo encarnado que ostenta como penacho una masa encefálica que pesa $1.5 \mathrm{~kg}$ y de la cual no hay en todo el mundo apenas 10 millones de Toneladas, lo que es bastante poco si recordamos que el recientemente reflotado Costa Concordia (que será un gran protagonista de esta historia de hundimiento y reinvención (pero no reflote) de la educación), pesaba “apenas" 114.500 Toneladas, el doble que el Titanic. Así, si rendimos un sentido homenaje (el mejor que podemos hacerle es estudiarlo a fondo), a su majestad el cerebro, es porque éste es el punto de partida y de llegada de todo aprendizaje, de cualquier estado vivido, de los estados de conciencia e inconsciencia, de nuestra felicidad y de nuestra perdición.

Dado que es nimia la diferencia anatómica o fisiológica entre el cerebro de los genios y de los normales como nosotros, de un

\section{De tal cerebro tal educación, y viceversa}

Lo mismo que ocurre con nuestro cerebro -que puede todo en potencia pero tan poco en acto-, ocurre con la educación. Los humanos somos aprendedores seriales, pero al mismo tiempo nos equivocamos en serie. La magia del aprendizaje de la lengua materna (reconstruida con un celo y una capacidad analítica excepcional por Deb Roy en The birth of a word), es una oda a la alegría del aprendizaje sin fin. Curiosamente es enorme e indescriptible lo mucho que cuesta el aprendizaje de una segunda lengua o de cualquier materia obligatoria, en comparación con la mos (velocidad de conexión, cantidad de conectados, ontología crowd, pensamiento en red) cada vez es más (y mejor) lo que hacemos en la red: pensamos en red, amamos en red, nos informamos en red, construimos en red, disfrutamos en red, criticamos en red, reinventamos en red, la lista sigue y sigue.

El misterio y el poder de la vida en red radica en un factor único y determinante, hacer es conectar y cuanto más y mejor conectados estemos, mejor será lo que podamos hacer. Steven Johnson lo dijo (citando a Louis Pasteur) en una pincelada genial "Chance favours the prepared mind", y ninguna mente esta mejor preparada que la mente en red. Por eso, ser en red es el formato más potente que podemos concebir para una ontología dinámica, fluida, generativa y básicamente social.

griego de la era de Pericles o un español de la era de Cervantes, de un atleta olímpico (figura muy alabada en el último TEDXTigre 2013), o un empleado de banco estándar, deberíamos preocuparnos por saber los motivos por los cuales padecemos todos los problemas globales que se han inventado monotemáticamente (pobreza, agresión, malnutrición, economías aniquiladoras, maltrato ambiental creciente, etc., etc.), sin que casi nunca se les halle una solución duradera.

Ello sobre todo porque el cerebro es infinitamente mucho más complejo, flexible y poderoso que las decisiones políticas (personales y colectivas) que él (cree) toma(r), las cuales son de una cortedad de miras, de un sectarismo sorprendente, y sobre todo de una autocomplacencia (que ignora el mal que con cada decisión autista le infligimos a otros y a nosotros mismos), que es resultado de nuestra miopía cognitiva, emocional y relacional.

fluidez y apropiación sin límites de la lengua materna.

Hay algo en el aprendizaje espontáneo (que tiene muy poco de genético, salvo la predisposición, y que necesita evidentemente de "triggers" y catalizadores externos para llegar a buen puerto), que no tiene el aprendizaje planificado o pedagógico, que despierta casi todos los "errores" del cerebro y muy pocas de sus maravillas.

La educación es mucho más compleja que las decisiones administrativas que toman los ministros, los supervisores, los inspec-

6 La suerte favorece a la mente preparada.

7 Disparadores que hacen que sucedan o se desencadenen acciones o sucesos deseables, previstos en su proceso más no en su resultado. 
tores, los directores de educación, los burócratas del aprendizaje y eso va más allá de las buenas intenciones. Cada declaración de lo que la educación debería ser (la pedagogía de la enunciación) va a contramano de lo que es (la pedagogía de la sorpresa y las preguntas), pero ante todo ignora los innumerables episodios de aprendizaje espontáneo, informal, cuasiautomático, inducidos por los pares, y especialmente por el deseo de aprender lo que cada uno necesita saber.

Hay un divorcio creciente entre la complejidad y la educación, del mismo modo que se presenta entre la tecnología y la política $y$, a menos que hilvanemos inconsutilmente estas cuatro dimensiones estratégicas del aprendizaje y del ser social significativo, seguiremos dilapidando fortunas en balas mágicas que ni siquiera son de plomo, muchas veces ni siquiera de arcilla, pero que tienen efectos no menos nefastos.

La cantinela de que debemos tener políticas de Estado, ignora cómo lograrlas; soñamos (o al menos eso aparece en las campañas políticas) con imitar a las ciudades inteligentes de Singapur (la primera en el rubro); con la creación de un sistema educativo como el finlandés, o con un crecimiento al nivel de las tasas chinas, cuando nosotros no somos (para bien y para mal) ni singapurenses, ni finlandeses, ni menos chinos, ni lo queremos ser.

Probablemente la dimensión más ignorada por los políticos sea justamente la de la complejidad (del aprendizaje) que se declina de infinitos modos; que está lejos de cualquier intención voluntarista, y puede transformar sociedades en pocas décadas (es el caso de los tres ejemplos antes mencionados), pero sólo en condiciones de continuidad, estabilidad, refuerzo y, sobre todo, mediante el uso constante e inteligente del cerebro, cosa que no vemos por nuestros lares.

Es por ello que si no educamos (en complejidad) a los políticos, no podremos politizar (en complejidad) a la educación, y viceversa. Porque sino politizamos la educación nunca tendremos una política de la complejidad, y por ahora vamos perdiendo en ambos rubros por goleada.

Estamos hartos de los finlandeses...

Mejor dicho, estamos hartos de que se invoque el ejemplo finlandés como encarnación de políticas de Estado vacuas y sim- plemente declamativas. Sabemos perfectamente bien por qué a los finlandeses les va tan bien como les va. Ello obedece a que:

- Son pocos.

- Saben demasiado.

- Enseñan por proyectos.

- Están sobrecalificados.

- Son antidisciplinarios.

- No creen en la tecnomagia.

Nosotros queremos ser finlandeses al uso nostro, es decir, sin que por estos pagos se dé ninguna de esas condiciones o constricciones, y aún así ser mejores que ellos. Dondequiera que vamos, adonde quiera que nos llaman (o nos hacemos llamar), trátese del tema de que se trate, llegamos sabiendo todo, y son muy pocos los que salen sabiendo algo.

En estas condiciones, para que podamos aprender mejor y distinto debemos deconstruir todo lo que tenemos. La política de los parches continuos (donde pendularmente atribuimos todos los males o todos los remedios a un factor causal alternativo y exclusivo: los (malos) docentes, los (pésimos) alumnos, las clases (atestadas), el pago (horrible), la falta de formación, el exceso de formación, el contagio maligno de los medios masivos, la falta de atención a la historia y a la memoria y, últimamente, la victimización que tiene nombre y apellido en las redes sociales: Facebook. Twitter, Foursquare, Whatsapp, pero sobre todo en Google como máquina de borrado de la atención y de imposición de un PageRank $k^{8}$ tendencioso de los recuerdos, nos ha hundido irreversiblemente.

El sistema educativo tal como existe es irrecuperable (como el Costa Concordia, ¿O no habría que rebautizarlo Costa Discordia?). Por ello, lo mejor que podemos hacer es reflotarlo y desguazarlo, operación que por otra parte tomó año y medio y costó la friolera de 600 millones de dólares. Ni scrapear algo grande sale barato en estos días.

Pero ojo, esta operación exige paradójicamente una capacidad de intervención minúscula, una estrategia laparoscópica mínimamente invasiva, una política de los toquecitos de mínima incisión y máxima potencia (como los innumerables cables que pudieron dar vuelta al paquidermo hundido). Reinventar el sistema educativo supone hackearlo casi todo al mismo tiempo:

8 Para el caso de Google, es el proceso (patentado) en que los algoritmos de búsqueda asignan relevancia a los objetos indexados, esta asignación depende, entre otros aspectos, del número y tipo o procedencia de los enlaces hacia un objeto (documentos, páginas). La consecuencia es el orden de despliegue a aparición de los enlaces a dichos objetos al realizar las búsquedas o consultas. 


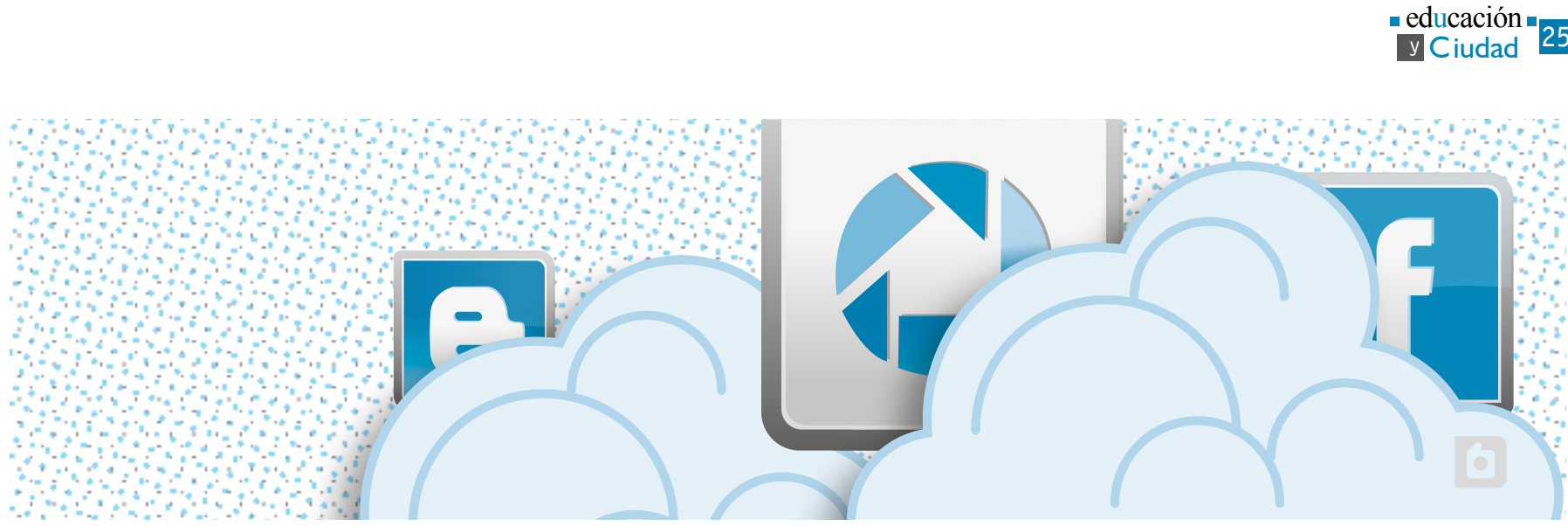

1. El espacio.

2. El tiempo.

3. La curricula.

4. La evaluación.

Con la misma intensidad y sin temor a perderlo todo por no querer quedarnos con casi nada de lo que teníamos (aunque al final será mucho lo que recuperaremos, pero esa es otra película).

\section{De la pedagogía de la enunciación a la pedagogía de la sorpresa (cerebridades)}

El origen de todos nuestros males pedagógicos está sobre diagnosticado, y aún así no queremos revisar ninguno de los supuestos de qué es lo que no anda, de la iatrogenia educativa 9 que nos lleva a multiplicar al infinito la creación de escuelas y de instituciones que no enseñan (como proclaman), pero que sobre todo no dejan aprender. Una de las aristas más peligrosas que han amplificado este juego de suma cero, es la ignorancia que la escuela (y la universidad) muestra hacia lo que la ciencia nos dice acerca de cómo se aprende, quiénes aprenden, cuándo se aprende y por qué se aprende.

La revolución neurocientífica ha quedado (como la de las redes sociales, la de la visualización de la información, la de inteligencia emocional, la de la ecología de los medios, la de la programación infantil masiva) a las puertas de la escuela.

¿Por qué ciencia y pedagogía están enfrentados a muerte? Si no nos hacemos esta pregunta (y no la resolvemos rápido), el actual estado de hambruna pedagógica alcanzará ribetes trágicos. $\mathrm{Y}$ aunque no nos tragaremos el anzuelo de las respuestas únicas, queda claro que la educación ignora elementos centrales de la biología del conocimiento: de que aprenden los cuerpos y no un cerebro aislado; de que saber es hacer y conectar prácticas significativas y no datos o huellas mnésicas desparramadas. Empecemos por dar sólo dos indicadores archiconocidos.

\section{Cuando el tiempo del cuerpo no coincide con el tiempo de los burócratas}

La cronobiología humana a la que el científico Diego Golombek (2003) le ha dedicado gran parte de su vida, muestra que los ritmos circadianos (una temporalidad intraorgánica) se sobreponen a los tiempos administrativos y burocráticos (los de la organización escolar). Para cada edad, en cada tiempo histórico y sobre todo en cada lugar, hay marcadores neuroquímicos que determinan nuestros estados de vigilia o de reparación cerebral.

El cuerpo adolescente, por ejemplo, no respeta la imposición de exigencias de aprendizajes definidos por una organización capitalista del mundo, que exige que los chicos estén en la escuela

9 Es decir, de aquellos males que la educación misma induce, por acción u omisión, en los estudiantes en formación. 
una hora antes de que los padres entren a su trabajo. Una de las consecuencia es que si en las primeras horas de la secundaria matinal no impartimos materias cercanas al cuerpo (deportes, actividades prácticas, arte, gestión, diseño), y en cambio las utilizamos coercitivamente para enseñar las materias de alta demanda cognitiva (biología, matemáticas, física, química, lengua), los resultados serán desastrosos. Como ocurre hoy en día.
Porque si bien el cerebro ama la repetición, no por ello renuncia a la diferencia, y si queremos que contenidos muchas veces emocionalmente neutros para los chicos, entren en su zona de resonancia y de interés, debemos empezar a tomarnos en serio la biología del conocimiento (siguiendo las huellas de Francisco Varela, Humberto Maturana, Heinz Von Foerster y, más recientemente, de Eric Kandel, Deb Roy y Sebastián Seung (2013).

\section{La importancia de la sorpresa como factor clave en el aprendizaje}

Trabajos recientes del equipo dirigido por Haydee Viola, donde sobresalen los aportes de Ballarini, et al. (2013), muestran que el grado de retención y de recuperación de experiencias y de recuerdos, cuando los mismos están acompañados por el factor sorpresa, explícitamente articulado como estrategia pedagógica, permiten mejorar y aumentar el aprendizaje en forma exponencial.

Más específicamente, la batería de experimentos demostró que experiencias novedosas mejoraron el recuerdo de actividades literarias o gráficas, especialmente si éstas tenían lugar al final de un ciclo de aprendizaje. Así hubo mejoras en la memoria entre grupos de estudiantes que habían asistido a una nueva clase de ciencia una hora antes o después de leer una historia, pero no así si entre ambos habían pasado más de 4 horas.

La promoción de efectos en la memoria de largo plazo tam-

bién se reprodujo con otro tipo de novedad, a saber, una clase de música y de otro tipo de aprendizaje que involucraba a la memoria visual. Como era de esperar, si la lección era conocida no logró incrementar el recuerdo de la tarea asociada. Para sorpresa de muchos, estos resultados confirman que acontecimientos nuevos relevantes durante las horas normales de la escuela, pueden mejorar la MLP de actividades aprendidas durante las horas normales de clase.

Dicho bien en castellano: nuevas formas de dar clase, nuevos ejemplos tradicionalmente ignorados, nuevas asociaciones y pautas que conectan y no figuran en el curriculum, ni forman parte de las estrategias pedagógicas convencionales, son justamente el "eslabón perdido" que hace falta, muy especialmente cuando tratamos con materiales complejos y de adquisición exigente (como es casi todo lo que importa ¿no?)

\section{Las profesiones de broadcast siguen copando la escena}

Cuando hace varios años atrás comenzamos nuestra cruzada denunciando a las profesiones P (Profesores, Printers, Publicistas, Periodistas y Políticos), a las que denominamos profesiones de broadcast (Piscitelli, 2011), sabíamos que la tarea no seria sencilla, que ninguno de sus cultores abandonaría fácilmente sus espacios atesorados, y que la reversión pedagógica (la puesta en práctica de clases dadas con la boca cerrada (Finkel, 2008), tendría resistencias inusitadas. No nos equivocamos, y vemos como todas las iniciativas por reformar la pedagogía existente son metabolizadas fácilmente por el sistema educativo, que termina invariablemente fagocitándose a la reforma.

Es por ello que no llama demasiado la atención que la publicidad siga siendo conductista, que a pesar de que críticos anti-consumo como Debord (2008) la hayan execrado hasta el paroxismo, aun así siga llevándonos felizmente al paraíso de la compra compulsiva de chatarra.
Otro tanto ocurre con los políticos, donde aunque la teoría de la aguja hipodérmica, según la cual la gente es fácilmente vacunada por los medios o por la palabra autorizada, ha sido deslegitimada desde hace décadas, creen que sólo se aplica a los otros emisores, pero no a ellos mismos. Ni qué decir del resto de la profesiones $\mathrm{P}$, y en especial de los docentes, que salvo en la estrambótica Finlandia no conmueven a casi nadie.

¿Por qué se supone que repetir indefinidamente algo significa que lo aprenderemos?, y mucho más aún, que sabremos cómo utilizarlo cuando más lo necesitemos. Por supuesto que el rote learning tiene valor de automatismo o de sedimento en ciertos casos muy particulares, trátese de las tablas matemáticas, la teoría o el solfeo musicales, la poesía y la letra de las canciones, la tabla periódica de los elementos o la lista de preposiciones gramaticales, y no mucho más. 
Pero salvo en ese terreno más que aceptado, la equivalencia entre enunciar e inyectar, sólo nos puede condenar a no ser escuchados, y a no mucho más. Hace más de un década que Don Finkel (2008) sistematizó la brillante idea de dar las clases con la boca cerrada, pero a excepción de los hipermediatizados Ken Robinson, Sugata Mitra, y el muchas veces citado pero rara vez encarnado, Jacques Jacotot, redescubierto por Jacques Ranciere y la eficiencia del maestro ignorante, no parece haber sobrepasado el nivel de entelequia o excepcionalidad que corresponde a los grandes nombres, muy lejos del aquí y ahora de los infinitos salones de clase que componen el mosaico de la educación realmente existente.

\section{Desfetichizar el espacio (habitar el conocimiento)}

Jeremy Bentham (1995) acuñó la metáfora espacial definitiva del panóptico, y de la mano de Michel Foucault primero, en la era analógica, y luego de cuestionadores como Evgeny Morozov, Arthur y Marilouise Kroeker, o Susanne Gaschke, la noción de lo digital era tantas veces vilipendiada como reinstalada en el centro de la atención pública como modelo de espacio execrable pero al mismo tiempo inevitable.

Hubo mentes benévolas que trataron de conciliar lo irreconciliable, por ello, la ergonomía, ya mucho tiempo atrás, de Frederick Winslow Taylor y Frank y Lillian Gilbreth, buscó introducir un poco de dulzura en el mundo esquizofrenizante del fordismo y el taylorismo, hasta llegar a los trabajos de Alphonse Chapanis. Por eso la ergonomía cognitiva de Donald Norman, a comienzos de los años 90, quiso llevar esa tregua al campo de las interaccio-

\section{El cuerpo (no es) un interfaz}

Hace tiempo que nos aburrió la comparación entre el quirófano de antaño y el del hoy (que supuestamente marcaría una distancia irreconciliable entre dos formas de practicar la medicina), de la misma forma que el ejemplo del espacio escolar que se mantendría intocado a lo largo de los siglos. Pero lo cierto es que la ocupación de las aulas en pleno 2013, sigue manteniendo invariantes una visión ortopédica del mobiliario, una sujeción atemporal del cuerpo, contradiciendo la propiocepción, y cualquier recorrido del cuerpo como interfaz, tal como hemos delineado en "ConectarLab" y que ha sido explorado también en el "MediaLab Eafit", y en los talleres de Catalina Quijano en la Universidad Tadeo Lozano.

Décadas de investigación y de empoderamiento restringido del cuerpo como interfaz, no logran perforar la certidumbre arquitectónica de una escuela entendida como disciplinamiento del alma. Y si bien la comparación del entorno escolar con las nes con las máquinas de pensar, que oscilan permanentemente entre el auxilio y el expolio.

Pero tantos devaneos, como felizmente dicen los brasileños, "non deu certo", y seguimos envueltos en cavilaciones absurdas cuando de repensar el espacio del aprendizaje se trata, y para qué hablar del laboral (que debería serlo aún más). Ocasionalmente aparecen obras de una potencia escandalizadora mayúscula, y si no, ¿qué otra cosa es ese mamotreto de Scott Doorley (2011)?, que parecería ser una oda a lo mal que marcha el espacio en casi todos lados, salvo cuando se decide intervenirlo con esos contraejemplos escandalosos (lo mismo ocurre con la arquitectura en general que es básicamente oprobiosa y que sólo se compensa con premios como el Pritzker, para las excepciones).

instituciones carcelarias, las instituciones totales, las instituciones voraces, las instituciones fordistas, las instituciones sin alma $O$ las instituciones antiergonómicas, es trivial, no por ello es falaz.

Lo que llama la atención no es tanto cómo la ciencia se ha puesto al servicio del disciplinamiento durante tanto tiempo, como la metáfora del panóptico lo ha reconocido con esmero, sino que numerosos ejemplos de ciencia al servicio del antidisciplinamiento, como tenemos el ejemplo de las escuelas Vittra, sean tan poco difundidos, pero mucho peor aún, escasamente imitados.

Quienquiera que haya transitado los espacios abiertos recorridos por una educación post-compulsiva, sabe de qué estamos hablando. Curiosamente, casi nadie conoce los esfuerzos de George Mesmin en Francia, y mucho menos los de Olga Cossettini en Argentina, para desanclar la arquitectura escolar de esa pesada carga simbólica y material.

\section{Del consumo compulsivo de contenidos a la}

La sentencia de Ludwig Feuerbach sigue filosofando a golpes de martillo: "somos lo que comemos, tanto material como simbólica-

\section{a producción no compulsiva de significado} mente"; sin embargo, el matiz que introduciremos no es menor: somos dónde comemos, material y simbólicamente también; confundir 
los espacios, comer en clase (como practicó María Acaso en la Complutense), llevar el living de su casa al espacio público de un $L a b$ (como viene de hacer Manuel Hernández, a quien acompañamos en el intento en "Plataforma Bogotá"), son intentos siempre insuficientes de trasmutar el orden coercitivo de la estandarización espacial y temporal.
Hace mucho tiempo, Luis F. Iglesias insistía en la afirmación de: "dime cómo organizas tu espacio y tu tiempo y te diré cómo enseñas". Este llamado nos llevará a otro elemento central en este proceso de desfetichización del tiempo (Algo que continuará con otros artículos y reflexiones).

\section{Nuevos lenguajes, nuevas competencias, nuevas inmersiones}

Hay una contradicción inevitable entre descreer del carácter deus ex machina de los artistas, atravesados como estamos por la ficción de que la inteligencia colectiva genera creadores a mansalva, y el recorrer intermitentemente la historia del arte, dejarnos atrapar por biografías de personajes impares (desde Velázquez y Goya, hasta Picasso y Dalí, desde Matisse y el aduanero Rousseau, hasta Paul Klee y Paul Cezanne, desde Marcel Duchamp y Jan Tinguely hasta George Braque y Vassily Kandisnky), que nos recuerdan inevitablemente la idiosincrasia de los creadores, la fuerza de su mirada disruptiva, la potencia de lecturas que perturban nuestra percepción y nuestra forma de recortar el mundo, ayudándonos, por fin, a ver lo mismo con ojos distintos.

Pero aunque seamos incapaces de reconciliar estas visiones antagónicas acerca de la fuerza creadora (¿será cierto que ya no hay pensadores, ni artistas, ni científicos, ni visionarios como antaño, y que el poder de romper con las miradas pasteurizantes ahora ya

\section{Tener más vs decir mejor}

Es obvio que disponer de la perspectiva no determina inevitablemente que habrá quienes la conviertan en un nuevo registro discursivo o en una semiótica no textual que modifique nuestra manera de ver el mundo, de interpretarlo y eventualmente de cambiarlo. Pero cualquier historia medianamente detallada de la interpenetración entre infraestructura estética y modos expresivos, revela que las mutaciones tecnológicas devenidas en nuevos lenguajes generalmente inventan a visionarios capaces de sacarles el jugo de una forma duradera y valiosa.

Porque hace décadas que contamos con diversas variantes de los entornos inmersivos. Desde las propuestas hoy ingenuas y tentativas de un Milton Krueger, en los años 90, hasta los desarrollos que nunca llegaron demasiado lejos de no está del lado de los individuos sino de los colectivos?), de lo que no cabe duda es que la ruptura de los marcos perceptivos requiere de un movimiento en dos momentos que incluye, por una parte, de cierta idiosincrasia, y por otra, de una ruptura equivalente de ciertas convenciones tecnológicas que sostienen esas miradas.

Por ello no cabe duda de que así como el mundo es muy diferente antes y después de Gutenberg, otro tanto ocurre con el mundo antes y después de la perspectiva, antes y después del puntillismo, el impresionismo, el constructivismo, el cubismo, y antes y después (he aquí el punto al que queríamos llegar) de las experiencias en entornos inmersivos, donde por inmersivo entendemos desde las formas más básicas de interactividad, hechas posibles por los lenguajes de programación, hasta las más extremas, que veremos encarnar prontamente y que fueron exploradas por Janet Murray, hipotéticamente, en Hamlet y el Holoverso (1997), y prospectivamente en The New médium (2011).

Jaron Lanier (y que él mismo criticaría inmisericorde en You are not a gadget, 2011), pasando por infinitas ediciones de ArtFutura (y de casi medio siglo de publicaciones en el Journal Leonardo), ha habido muchas más promesas que logros en el campo de la combinatoria entre efectos especiales, animación 3D, juegos estereoscopios y, sobre todo, usos estéticos de la realidad virtual. Con excepciones, ninguna de estas propuestas caló muy profundo en nuestra recalibración perceptiva, o en nuestra forma de abrevar en las historias, añadiendo sensorialidad y calidad háptica ${ }^{10}$ a las narrativas convencionales. No es lo mismo tener más fierros que hacer brillar el juego de las emociones. Es una perogrullada, pero ni críticos ni aduladores terminan (o empiezan) a entenderlo.

10 Que corresponde al mundo de las sensaciones más allá de las auditivas y visuales. 


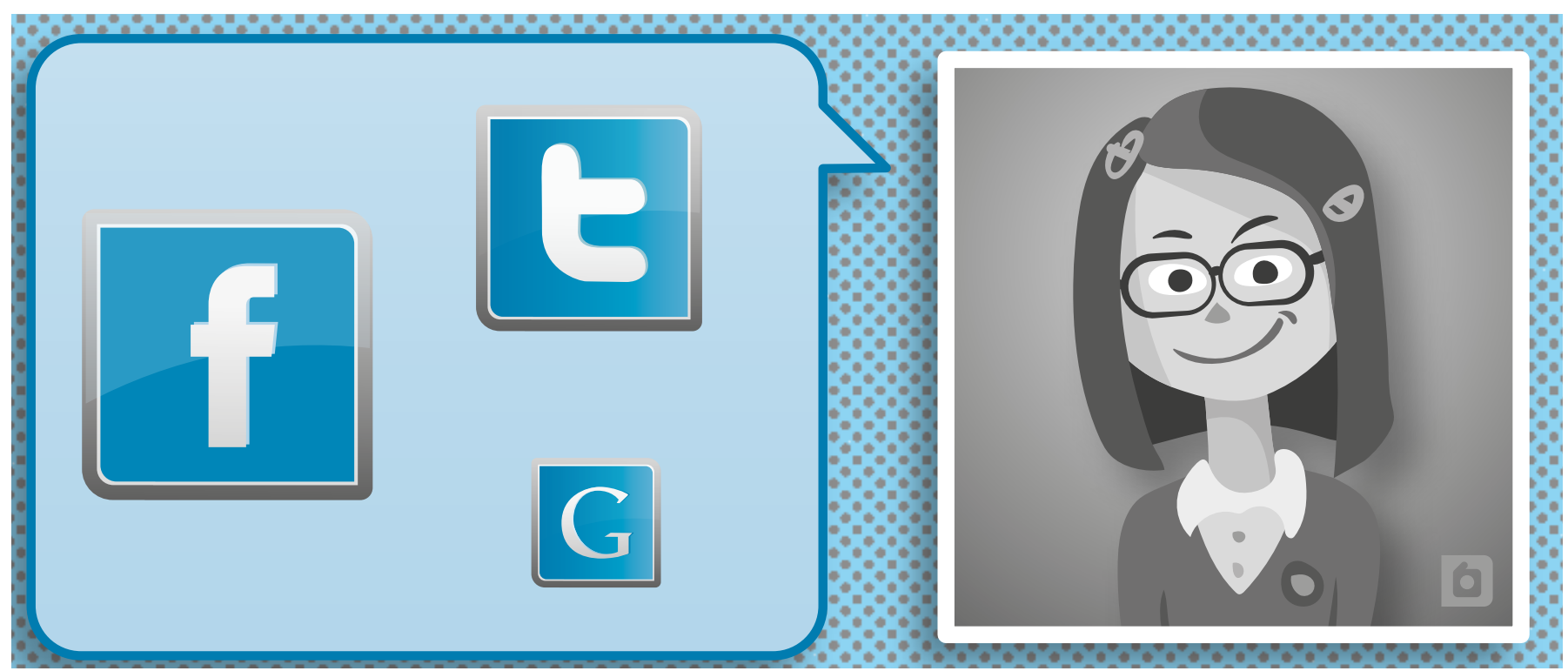

\section{Experiencias panorámicas en 360 grados}

Por eso cuando Maurice Benayoun (2011), un celebrado artista francés nacido en Argelia, quien publicó recientemente The dump: 207 bypothèses pour un passage à l'acte, nos invitó a visitarlo en su espacioso despacho que da a una bella vista en Kowloon, a pocas cuadras del metro Kowloon Tong, y nos ofreció un poco del sabor de lo que se está haciendo en el Run Run Shaw Creative Media, visitando la Gallery 360, supusimos que se trataría de algún show o espectáculo potencialmente interesante, pero no mucho más.

Error, "as usual", como pasa con tantos prejuicios. Porque con Benayoun se trata de una experiencia inmersiva que abre la participación, a visitar centenares de templos y grutas budistas tapizadas de murales, estatuas y monumentos. Hay cerca de 700 cuevas en la zona, de las cuales cerca de 500 contienen murales y esculturas de gran valor. Las cuevas de los mil budas están situadas en Dunhuang,

\section{Pure Land. Inside the Mogao Grottoes at Dunhuang}

En el caso que nos tocó vivir se trató de una visita en profundidad a una historia pintada en la pared norte de la cueva 220, bautizada como Paraíso Oriental de Bhaisajyaguru, el médico de Buda. Muestra las siete formas o emanaciones que el médico puede tomar como curador. Se trata de una fila de figuras encastradas en plataformas de lotos, con una fuente debajo acompañada de numerosos músicos que asisten a cuatro bailarines. La historia cuenta los 12 grandes votos de Buda en relación a la comida, la un pueblito en el noroeste chino, en medio del desierto de Gobi.

Mientras duró la ruta de la seda, entre el siglo II a.C., y el siglo XIV de la era cristiana, se trató del camino privilegiado entre Oriente y Occidente, y como sabemos las cuentas y los cuentos van juntos desde tiempo inmemorial, aunque no les guste a muchos. Usando tecnologías de 3D, los tecnoartistas de la City University of Hong Kong lograron que los visitantes nos sumerjamos en una experiencia panorámica en 360 grados que nos devuelve la sensación imposible (las cuevas más importantes han sido selladas, la única iluminación que alguna vez tuvieron fue a través de una linterna de baja potencia focalizada en algún detalle) de estar dentro de las cuevas en una escala 1 a 1 . En el camino, las figuras y los objetos son animados, se les ha superpuesto sonido y se han recreado los colores originales de las representaciones.

bebida, la ropa, la medicina y el mundo espiritual. Los devotos testimoniaban su adoración iluminando lámparas votivas.

Lo que vimos durante una hora es un prototipo en el que colaboraron 30 personas durante 6 meses, y es de una intensidad muy llamativa. El uso de una consola de PlayStation para navegar el espacio, es un ejemplo de tecnología aumentada bien empleada. La lupa que pone en foco los detalles vistos en 3D es de un realismo inusitado. La expectativa que Holland Cotter (2008) 
había planteado en su nota Budda's Caves, hace 5 años, ha tenido un final feliz.

Se trata de una solución remedial frente a un problema que no tiene solución: ¿cómo preservar estos tesoros amenazados por siglos de humedad y de corrosión natural, que ahora son subsi-

\section{¿Multimedia o metamedia?}

Como estrategia es apropiada, pero como efecto logrado inmejorable. Claro que aquí también los conceptos que utilicemos cuentan. Porque a diferencia de muchas otras experiencias en 3D que resultaron más de lo mismo, en este caso a lo que estamos asistiendo es a una experiencia nueva, a un aumento cognitivo de las tecnologías (en el mejor sentido de Douglas Engelbart, 1962), a una experiencia 3D visual que renueva el drama estético, narrativo y espiritual, de esas pinturas y esculturas hasta hace poco encapsuladas en esas cuevas, invisibles y desconocidas para todos.

Lo que PureLand logra, es redefinir qué entendemos por preservación digital, por interpretaciones de la herencia cultural, y qué primeros pasos está dando la museografía enactiva para lograrlo, con las inmensas consecuencias educativas que ello supone. Aunque exageradamente cara, circunscripta a espacios como esta galería (gracias a las astutas intuiciones y realizaciones del director del centro Jeffrey Shaw y de Sarah Kenderdine), lo que vemos aquí es una feliz puesta en escena de las hipótesis de James Cameron y de Alfonso Cuarón en otro contexto, quien sostiene orondo que:

"Cuando ves una película concebida con 3-D en la mente, realmente puede volar tu cabeza. Es una herramienta creativa de la que muy pocas personas se han aprovechado. Se la puede utilizar para hacer una experiencia inmersiva, que es lo que teníamos que hacer aquí: el espectador tenía que guientemente depredados por la pulsión escópica de los turistas? Una posibilidad como se hizo en Altamira y Lascaux, es crear réplicas presenciales, la otra es utilizar fotografías de alta resolución y el escaneo láser en 3D, para poder recrear una experiencia imposible de vivir en el espacio físico real.

estar con Sandra mientras flota en el espacio, tenía que estar allí con ella cuando los escombros están volando hacia ella. Para mí, Gravedad en 2-D es sólo 20 por ciento de la experiencia"

El primero en Avatar y el segundo en Gravity (pero también otras obras como Pina, Cave of Forgotten Dreams, Life of $P$ ) , han llevado al cine historias épicas de colectivos, uno y de individuos, el otro (con una Sandra Bullock inesperadamente dramática, viril y sobre todo resiliente), potenciando la narrativa a partir de un 3D creíble y redivivo.

Otro tanto ocurre con PureLand, uno de los chiches $^{11}$ más exitosos del Creative Center que sienta precedentes en una nueva forma de explorar los medios y puntuar la realidad. Como la Gallery 360, es un simulador de propósito general, en nuestra visita pudimos apreciar dos intervenciones más.

Por fin estamos viendo algunos ejemplos pioneros no ya de multimedios, sino de metamedios. Qué sea este formato o género, y cómo se puede potenciar de ahora en más, es algo que Lev Manovich (2013) y otros pioneros están ayudando a delinear. Si queríamos muestras de cómo los nuevos lenguajes, combinados con las estrategias anti y post-diciplinarias -y profundamente $e d u$ punks (Acaso, 2013)-, están ayudando a reintentar la educación, estos ejemplos lo corroboran en demasía.

11 Artilugio atractivo que por extensión alude a juguetes (nota del editor). 


\section{Referencias}

Acaso, María (2013). rEDUvolution. Hacer la revolución en la educación. Madrid: Paidós.

Alvarado, Rafael. (2011). The Digital Humanities Situation. Obtenido desde http://transducer.ontoligent.com/?p=717

Ballarini, F., Martínez, M.C., Díaz Perez, M., Moncada, D., y Viola, H. (2013). "Memory in Elementary School Children Is Improved by an Unrelated Novel Experience”. PLoS ONE, 8(6), e66875. doi:10.1371/ journal.pone.0066875.

Benayoun, M. (2011). The dump: 207 hypothèses pour un passage à l'acte. USA: FYP Editions.

Bentham, J. (1995). The Panopticon Writings. Londres: Verso..

Berry, D. M. (Ed.). (2012). Understanding Digital Humanities. Londres: Palgrave Macmillan.

Buchanan, M. (2003). Nexus: Small Worlds and the Groundbreaking Theory of Networks. New York: W. W. Norton \& Company.

Christakis, N., y Fowler, J. (2010). Conectados. El sorprendente poder de las redes sociales y cómo nos afectan. Barcelona: Taurus.

Cotter, Holland. (2008, Julio 6). Buddha’s Caves. New York Times.

Debord, Guy. (2008). La sociedad del espectáculo. Sevilla: Edición Doble J.

Doorley, Scott, et al. (2011). Make Space: How to Set the Stage for Creative Collaboration. New York: Willey \& Sons.

Engelbart, Douglas (1962). Augmenting buman intellect: A conceptual framework. SRI Summary Report AFOSR-3223.

Finkel, D. (2008). Dar la clase con la boca cerrada. Valencia: Universidad de Valencia.

Gold, M. K. (Ed.). (2012). Debates in the Digital Humanities. Minnesota: University of Minnesota Press.
Golombek, Diego. (2003). Cronobiologia bumana en busca del tiempo perdido. Ritmos y relojes biológicos en la salud y en la enfermedad. Buenos Aires: Prometeo.

Lanier, J. (2011). You are Not a Gadget: A Manifesto. New York: Vintage Books.

Laszlo Barabasi, A. (2002). Linked: The New Science Of Networks Basic Books. New York: Perseo.

McCarty, Willard. (2005). Humanities Computing. Basingstoke. UK: Palgrave Macmillan.

Manovich, Lev. (2013). Software Takes. Command. Londres: Bloombsbury Academic

Murray, Janet. (1997). Hamlet on the bolodeck. The future of narrative in cyberspace. New York: New York Free Press.

Murray, Janet. (2011). Inventing the Medium: Principles of Interaction Design as a Cultural Practice. Massachusetts: The MIT Press.

Piscitelli, Alejandro (2011). El Paréntesis de Gutenberg. La religión digital en la era de las pantallas ubicuas. Buenos Aires: Santillana.

Ramsay, Stephen (2011). Reading Machines: Toward an Algorithmic Criticism. Illinois: University of Illinois Press.

Schreibman, S. S., y Ray Unsworth, J. (Ed.). (2008). A Companion to Digital Humanities. Oxford: Wiley-Blackwell.

Seung, Sebastian. (2013). Connectome: How the Brain's Wiring Makes Us Who We Are. New York: Mariner Books.

Solé, Ricardo. (2009). Redes Complejas, del Genoma a Internet. Barcelona: Tusquets.

Watts, D. J. (2004). Six Degrees: The Science of a Connected Age. W. W. New York: Norton \& Company. 
$\infty$ 